\title{
Multiple Intraventricular Non-Hodgkin Lymphoma - A Rare Case Report
}

\author{
Hossain $\mathrm{MN}^{1}$, Rashid $\mathrm{MH}^{2}$, Mamun $\mathrm{AA}^{3}$, Hasan $\mathrm{M}^{4}$
}

Conflict of interest: There is no conflict of interest relevant to this paper to disclose.

Funding Agency : was not funded by any institute or any group.

Contribution of Authors: Principal Investigator- Dr. Mohammad Nazrul Hossain

Manuscript preparation- Dr. Mohammad. Humayun Rashid

Data collection- Dr. Abdullah Al Mamun

Editorial formatting- Dr. Mahmudul Hasan

Copyright: @2020bang.BJNS published by BSNS. This article is published under the creative commons CC-BY-NC license. This license permits use distribution (https://creativecommons. orgf/licences/by-nc/4-0/)reproduction in any medium, provided the original work is properly cited and is not used for commercial purposes.

Received: 21.06 .2020

Accepted: 01.10.2020

\begin{abstract}
:
Lymphoma of the central nervous system can present with a wide variety of clinical symptoms and imaging characteristics which can be challenging for radiologist as well as neurosurgeons to deal with. Primary CNS lymphoma (PCNSL) accounts for $1 \%$ of all primary brain tumors which is a rare variant of extranodal non-Hodgkin's lymphoma. We report a case primary central nervous system Non-Hodgkins lymphoma presenting a multiple intraventricular and periventricular masses in a 14 years young man with severe headache, convulsion and unsteady gait. The diagnosis was obtained by image guided surgical biopsy. This is an unusual presentation of primary central nervous system lymphoma involving lateral ventricle and $3^{\text {rd }}$ ventricle while the majority of the case presenting as solitary masses.
\end{abstract}

Keywords: CNS Lymphoma, PCNSNHL, Brain Tumor, Non-Hodgkin Lymphoma

Bang. J Neurosurgery 2021; 10(2): 210-215

\section{Introduction:}

Lymphomas are heamotological malignancies where extranodal manifestations in approximately $40 \%$ cases. ${ }^{1}$ Central nervous system lymphoma can be divided into Primary CNS lymphoma (PCNSL) and Secondary CNS lymphoma where Secondary CNS lymphoma is generally considered as CNS involvement in lymphoma that was not evident at the initiation of treatment for systemic lymphoma outside the CNS. ${ }^{2,3}$ Primary CNS lymphomas (PCNSL) are aggressive malignancies that arise in distinct anatomical sites, which display unique structural, biological and immunological conditions and accounts for $1 \%$ of all primary brain tumors. So far, despite recent therapeutic advances, these malignancies exhibit one of the worst prognoses among all non-Hodgkin lymphomas (NHL). ${ }^{4} \mathrm{PCNSL}$ lesions are virtually always found within the brain parenchyma and may be superficial (subpial) or deep-seated (sub ependymal) but Intraventricular PCNSL is an extremely rare presentation of PCNSL with $=15$ patients described in literature. ${ }^{5}$

1. Dr. Mohammad Nazrul Hossain, Associate Professor \& Head, Department of Neurosurgery, Ibrahim Cardiac Hospital \& Research Institute, Dhaka, Bangladesh.

2. Dr. Mohammad. Humayun Rashid, Assistant Registrar, Department of Neurosurgery, Ibrahim Cardiac Hospital \& Research Institute, Dhaka, Bangladesh.

3. Dr. Abdullah Al Mamun, Resident Medical Officer, Department of Neurosurgery, Ibrahim Cardiac Hospital \& Research Institute, Dhaka, Bangladesh.

4. Dr. Mahmudul Hasan, Resident Medical Officer, Department of Neurosurgery, Ibrahim Cardiac Hospital \& Research Institute, Dhaka, Bangladesh.

Address of Correspondence: Dr. Mohammad Nazrul Hossain, Associate Professor \& Head, Department of Neurosurgery, Ibrahim Cardiac Hospital \& Research Institute, Dhaka, Bangladesh. Phone: 01819496333. E-mail: drmnh2003@gmail.com. 


\section{Case Report:}

A young 14-year-old boy had a 2 months history of continuous headache, recurrent vomiting, and multiple episodes of generalized tonic-clonic seizure. The patient described his headache as generalized, slightly dull, not persistent and without any exacerbating or relieving factors. He denied fever, dizziness but complaints of nausea or vomiting more marked at morning than rest of the day and it was aggravated by coughing or bending forward and relieved by lying down. He also had history of one episode of convulsion which started from the left hand and gradually progress to generalized tonic-clonic seizure. The convulsion persisted for 2-3 minutes. Convulsion was not associated with any prodrome or aura. It was not also associated with frothing or tongue bite or loss of consciousness or bowel bladder incontinence and no post ictal amnesia. This similar type of convulsion occurs for 2-3 times during the course of his illness. There was slight unsteady gait was noted He had no ocular complaints such as blurred vision, floaters, decreased acuity, pain, photophobia and diplopia. There was also no history of cognitive decline and personality changes, loss of coordination and immunosuppressive drug intake, radiation exposure, and systemic infection or other autoimmune diseases. His family history was unremarkable. Neurologic examination did not show any focal neurological signs. Contrast CT scan of Brain demonstrated moderately enhancing multiple ependymal and sub ependymal mass lesion in and around frontal and temporal horn, body of right lateral ventricle. Similar lesion also seen in mid sub callosal region, infront of $3^{\text {rd }}$ ventricle and left occipital horn. Associated ventricle near masses are compressed with dilatation of rest of the lateral ventricle. Mild perilesional oedema was present. Contrast MRI depicts that multiple (about 6 in number) well demarcated round / oval shaped variable sized lesions, larger one measuring about $3.03 \mathrm{~cm} \times 1.85$ $\mathrm{cm}$ which are almost isointense to gray matter on both $\mathrm{T} 1$ and $\mathrm{T} 2 \mathrm{WI}$, mild perilesionla oedema, compression / invasion of lateral, $3^{\text {rd }}$ ventriclesm mild shift to left, mild ventricular dilatation with mild diffusion restriction in Diffusion-weighted imaging. After contrast, inhomogenous solid, ring enhancement of the lesions with periventricular ependymal enhancement was noted. Axial SWI demonstrates enlarged veins in the thalamus and located adjacent to the lateral ventricles. The patient was started with Dexamethasone and RT PCR for COVID-19 test was done. After 2 sample were negative, patient underwent image guided surgical biopsy from body of right lateral ventricle. After adequate sample for biopsy was taken, external ventricular drainage was applied through the same burr hole and secured. Biopsy revealed a neoplasm composed of round cells arranged diffusely. These cells have hyperchromatic contorted nuclei with prominent nucleoli and scant cytoplasm. Mitoses and apoptotic debris present, The histologic appearance is that of a small round cell tumor, consistent with Non-Hodgkin lymphoma.

Patient experienced a complicated hospital course after biopsy. There was delayed recovery from anaesthesia although Aldrete and Kroulik modified post anesthetic recovery score was 7 (Consciousness-1, Aactivity-1, Respiration-1, Circulation-2, Peripheral oxygen saturation-2) out of 10 at 30 mins post operatively. There was new onset atrial fibrillation and episode of status epilepticus leading to intubation 6 hours after surgery. Immediately CT scan of Brain was done and it revealed intraventricular hemorrhage although external ventricular drainage was in situ predicting post procedure ventricular hematoma. Sadly patient experienced cardiac arrest and had expired on $3^{\text {rd }}$ post operative day.
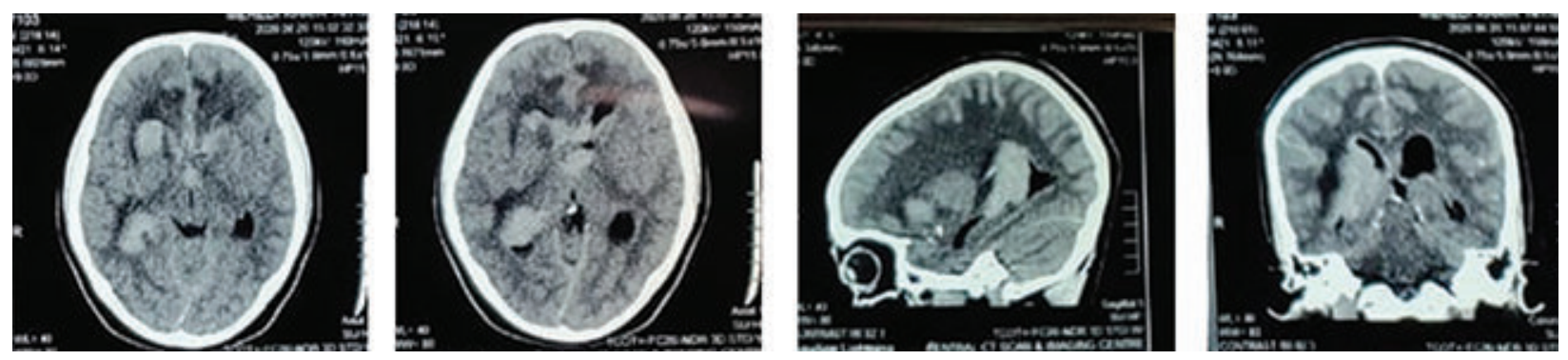

Fig.-1: 
Contrast CT Scan : Contrast CT scan of Brain demonstrated moderately enhancing multiple ependymal and sub ependymal mass lesion in and around frontal and temporal horn, body of right lateral ventricle. Similar lesion also seen in mid sub callosal region, infront of $3^{\text {rd }}$ ventricle and left occipital horn. Associated ventricle near masses are compressed with dilatation of rest of the lateral ventricle.

Figure: Contrast MRI (A-I) depicts that multiple (about 6 in number) well demarcated round / oval shaped variable sized lesions, larger one measuring about $3.03 \mathrm{~cm} \times 1.85 \mathrm{~cm}$ which are almost isointense to gray matter on both $\mathrm{T} 1$ and $\mathrm{T} 2 \mathrm{WI}$, mild perilesionla oedema, compression / invasion of lateral, $3^{\text {rd }}$ ventricles, mild shift to left, mild ventricular dilatation with mild diffusion restriction in Diffusion-weighted imaging. After contrast, inhomogenous solid, ring enhancement of the lesions with periventricular ependymal enhancement was noted. Axial SWI demonstrates enlarged veins in the thalamus and located adjacent to the lateral ventricles.

Figure: Image guided ( Stealth Neuro-navigation sytem ) surgical biopsy (A, B) was done with passive biopsy needle using target alignment error at or below $1.6 \mathrm{~mm}$ accuracy and external ventricular drainage ( C ) was applied with navigation guide universal navigus probe.

Figure: Histopathology slide revealed a neoplasm composed of round cells arranged diffusely. These cells have hyperchromatic contorted nuclei with prominent nucleoli and scant cytoplasm. Mitoses and apoptotic debris present, The histologic appearance is that of a small round cell tumor, consistent with Non-Hodgkin lymphoma.
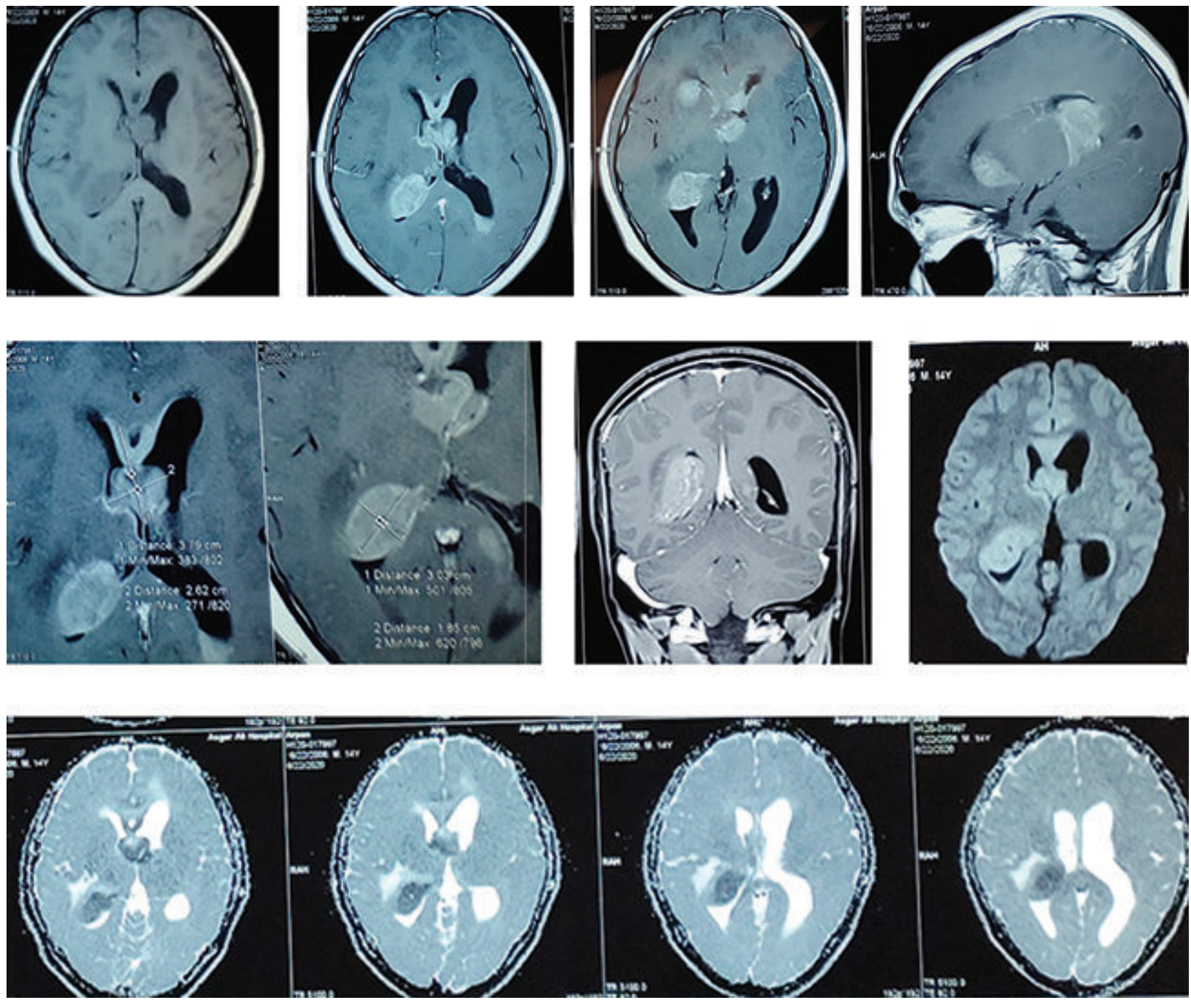

Fig.-2: 

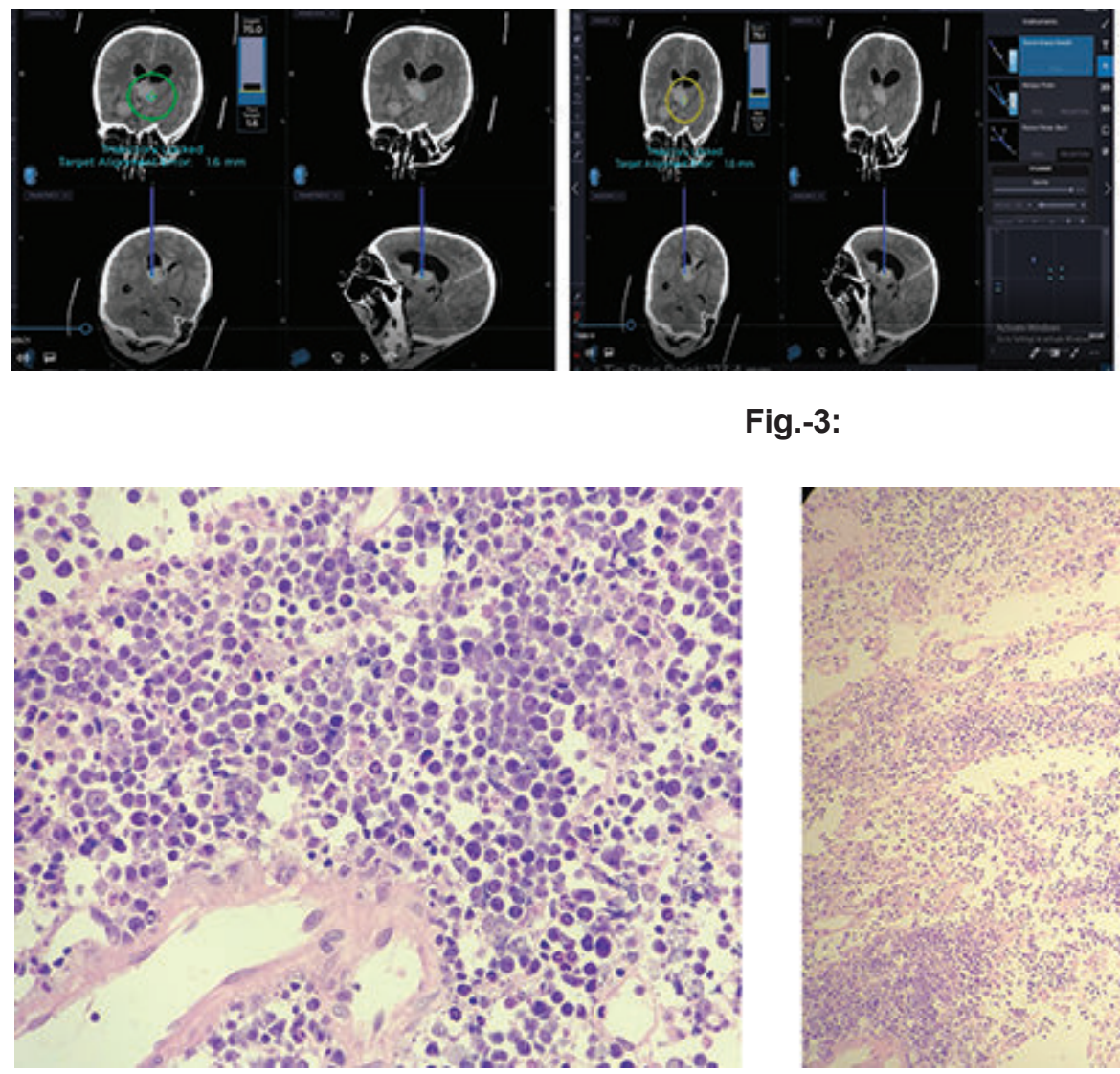

\section{Discussion:}

To our knowledge, this is the first reported case of primary central nervous system Non-Hodgkin lymphoma involving lateral ventricle, $3^{\text {rd }}$ ventricle and periventricular area. PCNSL was most often seen either in the setting of known immuno compromised status or with advanced age before the emergence of HIVIAIDS. After emergence of the AIDS epidemic was mirrored by a rise in the incidence of PCNSL, and it soon became an AIDS defining illness. ${ }^{6}$ Most of the PCNSL cases present as solitary masses in cerebral hemisphere, basal ganglia, thalamus, and periventricular region. Only a few cases of PCNSL involving ventricular system have been reported in the literature. CNS lymphomas are highly chemo and radiation sensitive tumors and management of these tumors is histopathological confirmation by either open or stereotactic biopsy followed by chemotherapy or radiotherapy or both. ${ }^{6,7}$ Primary intraventricular lymphomas are rare entity. 7,9 Only a few cases of intraventricular PCNSL have been reported in the literature, of which three cases were in the fourth ventricle, ${ }^{[10,11]}$ four cases were in the third
Fig.-3:

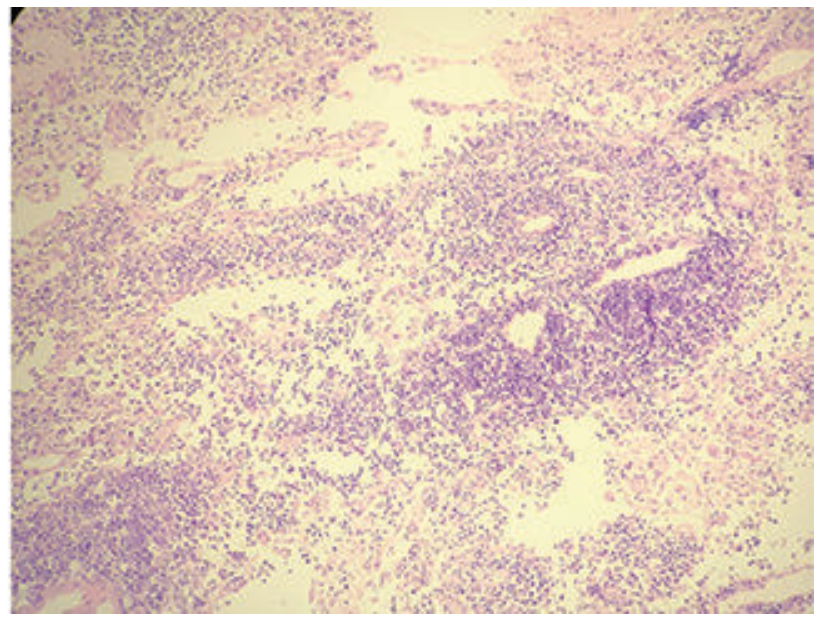

Fig.-4:

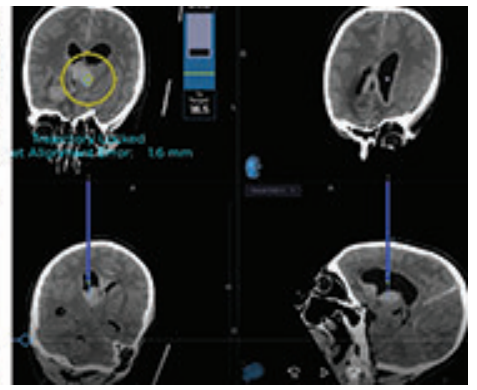

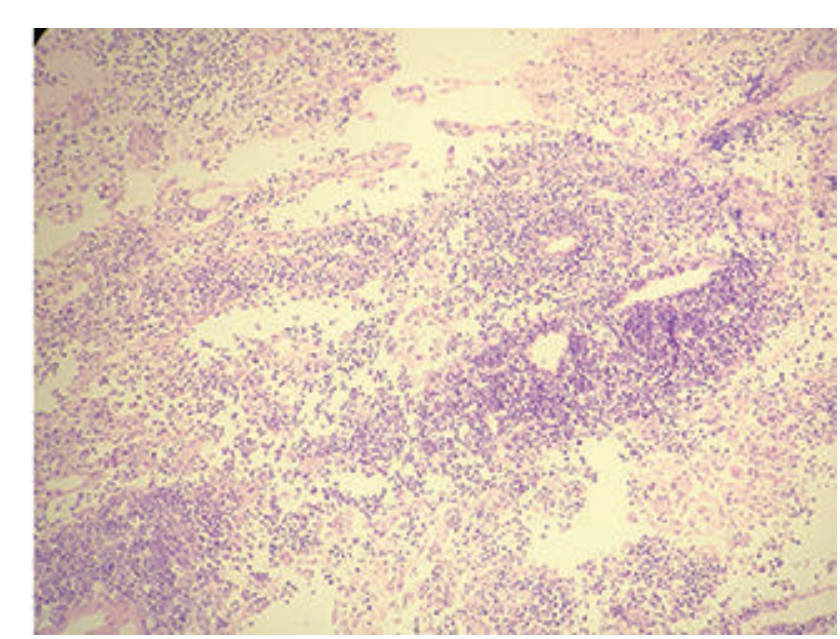

ventricle. ${ }^{12,13}$ and three cases were in the lateral ventricle. ${ }^{14,15}$ while only one case as per literature available was identified with simultaneous involvement of the lateral and fourth ventricles.

The imaging characteristics of PCNSL are created by the hypercellularity of the tumor and its high nuclear to cytoplasmic ratio. ${ }^{16}$ Just as our patient's initial CT showed a hyperdense lesion, classically, the lesions appear as hyperdense or isodense on non contrasted $\mathrm{CT}$. The hyperdensity is nonspecific and can lead the incorrect consideration of hemorrhage within the lesion. Typical Hounsfield unit for acute hemorrhage is 6090. However, the hyperdense appearance on CT can also help distinguish the tumor from metastasis and gliomas which tend to be more hypodense. With contrast enhanced CT, these lesions almost always demonstrate enhancement due to disruption of the blood brain barrier.

On MRI, the typical appearance of PCNSL is hypointense to isointense on T1-weighted images (T1WI) and isointense to hyperintense on T2weighted images (T2WI). In non AIDS patients, there 
is almost always homogenous contrast enhancement $(90 \%)$ and it is uncommon to see ring enhancement. With the AIDS population, contrast enhancement is more irregular and ring enhancement may be seen in up to $75 \%$ of cases ${ }^{[16]}$. PCNSL is sometimes referred to as a "ghost tumor" because corticosteroid administration has been reported to cause lesions to disappear or lose their contrast enhancement. ${ }^{17}$

Mass effect tends to be mild to moderate despite occasional significant edema surrounding the tumor. ${ }^{16-}$ ${ }^{17}$ Diffusion weighted imaging (DWI) can also provide additional information. Due to the highly cellular nature of these tumors, there is often restricted diffusion making the lesions appear high intensity on DWI and hypointense on apparent diffusion coefficient (ADC). Our patient's tumor demonstrated mild restricted diffusion. Unfortunately, restricted diffusion itself is very nonspecific for PCNSL and can be seen with multiple other disease processes.

The top differential for intraventricular CNS lesions includes central neurocytoma, meningioma, ependymoma, choroid plexus papilloma, and metastasis. ${ }^{18}$

Although the occurrence of PCNSL is extremely rare and can be present in many different forms, including the possibility of such tumor in the differential diagnosis of intraventricular neoplasms is required, particu-larly in cases where the lesion is located in one or multiple ventricles and exhibits a cluster like appearance [19]. Surgical resection is not an efficient treatment method for PCNSL unless urgent surgical decompression is required; however, the disease may be controlled by systemic chemotherapy or intrathecal chemotherapy and radiotherapy. ${ }^{20}$ Therefore, only a limited biopsy is required to confirm the diagnosis of a lymphoma, thereby preventing serious after effects.

\section{Conclusion:}

Primary central nervous system lymphoma (PCNSL) can have a variety of imaging characteristics which are non specific but can aid in distinguishing this lesion from others encountered within the central nervous system, such as the intraparenchymal and supratentorial location and typical lack of necrosis, hemorrhage, cystic appearance, and ring enhancement (except in autoimmune deficiency syndrome (AIDS)- related PCNSL). Although advanced imaging techniques can increase the diagnostic accuracy and help in differentiating PCNSL from other tumours or non-tumour intraventricular lesions such as central neurocytomas, meningiomas, ependymomas, choroid plexus papilloma, metastasis, neurocysticercosis but the radiologist needs to communicate PCNSL in the differential diagnosis so clinicians can obtain tissue via brain biopsy or cerebrospinal fluid cytology as PCNSL remains a histologic diagnosis. Therefore, the standard treatment for PCNSL is biopsy followed by systemic chemotherapy or with intrathecal chemotherapy.

\section{Reference:}

1. Gigli S, Buonocore $\mathrm{V}$, Barchetti F, et al. Primary colonic lymphoma: An incidental finding in a patient with a gallstone attack. World J Clin Cases 2014;2:146-50.

2. Baraniskin A, Deckert M, Schulte-Altedorneburg G, Schlegel U, Schroers R. Current strategies in the diagnosis of diffuse large B-cell lymphoma of the central nervous system. Br J Haematol 2012;156:421- 32.

3. Tomita N, Kodama F, Kanamori H, Motomura S, Ishigatsubo Y. Secondary central nervous system lymphoma. Int $J$ Hematol 2006;84:128-35.

4. Andr'es J.M.F, Michele Reni. Primary central nervous system lymphoma. Critical reviews in oncology hematology 2007:63:257-268

5. Vinit S, Venkatesh M, Manish K, Kaushal P, Sogani S. $\mathrm{K}$, Kunal S. Primary intraventricular central nervous system lymphoma in an immunocompetent patient. Journal of Pediatric Neurosciences 2015: 10(4):393-395

6. Kumar H, Sharma A, Sharma V, Singhvi S. Primary central nervous system lymphoma involving entire ventricular system. Asian J neurosurg 2020; 15:126-7. doi: 10.4103/ ajns.AJNS_94_16

7. Cecchi PC, Billio A, Colombetti V, Rizzo P, Ricci UM, Schwarz A. Primary high grade B cell lymphoma of the choroid plexus. Clin Neurol Neurosurg 2008;110:75 9.

8. Park SW, Yoon SH, Cho KG. An endoscopically proven ventriculitis type, cyst like intraventricular primary lymphoma of the central nervous system. Acta Neurochir (Wien) 2006;148:981 4.

9. Bühring $U$, Herrlinger $U$, Krings T, Thiex R, Weller M, Küker W. MRI features of primary central nervous system lymphomas at presentation. Neurology 2001;57:393 6 .

10. Haegelen C, Riffaud L, Bernard M, Morandi X. Primary isolated lymphoma of the fourth ventricle: Case report. J Neurooncol 2001;51:129 31.

11. Hill CS, Khan AF, Bloom S, McCartney S, Choi D. A rare case of vomiting: Fourth ventricular B cell lymphoma. J Neurooncol 2009;93:261 2. 
12. Kelley TW, Prayson RA, Barnett GH, Stevens GH, Cook JR, Hsi ED. Extranodal marginal zone B cell lymphoma of mucosa associated lymphoid tissue arising in the lateral ventricle. Leuk Lymphoma 2005;46:1423 7.

13. Jung TY, Jung $\mathrm{S}$, Lee MC, Lee $\mathrm{KH}$. Extranodal marginal zone $B$ cell lymphoma mimicking meningioma in lateral ventricle: A case report and possible pathogenesis. J Neurooncol 2006;80:63 7.

14. Terasaki M, Abe T, Tajima Y, Fukushima S, Hirohata M, Shigemori M. Primary choroid plexus T cell lymphoma and multiple aneurysms in the CNS. Leuk Lymphoma 2006;47:1680 2

15. Bogdahn U, Bogdahn S, Mertens HG, Dommasch D, Wodarz R, Wünsch $\mathrm{PH}$, et al. Primary non Hodgkin's lymphomas of the CNS. Acta Neurol Scand 1986;73: 60214.

16. Haldorsen IS, Espeland A, Larsson EM. Central Nervous System Lymphoma: Characteristic Findings on Traditional and Advanced Imaging. Am J Neuroradiol 2011;32:98492. PMID: 20616176.

17. Yap KK, Sutherland T, Liew E, Tartaglia CJ, Pang M, Trost N. Magnetic Resonance Features of Primary Central Nervous System Lymphoma in the Immunocompetent Patient: A Pictorial Essay. Journal of Medical Imaging and Radiation Oncology 2012;56:179-186. PMID: 22697326.

18. Funaro K, Katie CB, Sadie A, Steven JA3, Christopher V. A Case of Primary central nervous system lymphoma.Radiology Case. 2014 Mar; 8(3):1-8. doi: 10.3941/jrcr.v8i3.1361

19. Lettau $M$ and Laible M: Primary intraventricular non Hodgkin's lymphoma of the CNS. Rofo 2012;184: 261263 (In Chinese)

20. Jia-Zhen Q, Yue-Kui W, Zhi-Jun Y, Jun LV, Yuan-Yuan D, Hong-Tian Z, Yi-Wu D. Endoscopic biopsy of B cell lymphoma involving the entire ventricular system : A case report. Experimental and therapeutic medicine. 2016;11:325-327 